\title{
Nasal Eosinophilia in Allergic Rhinitis: A Clinico-Correlative Observational Study
}

\author{
Gupta $\mathrm{S}^{1}$, Belaldavar $\mathrm{BP}^{2}$ and Havaldar RR ${ }^{2 *}$ \\ ${ }^{1}$ Consultant Surgeon, Doon ENT Hospital, India \\ ${ }^{2}$ Department of ENT and Head \& Neck Surgery, JN Medical College, India
}

*Corresponding author: Rajesh R Havaldar, Department of ENT and Head \& Neck Surgery, JN Medical College, Karnataka, India, Tel: +917090095006; Email: rajeshhavaldar@yahoo.com

\section{Research Article}

Volume 5 Issue 1

Received Date: May 21, 2020

Published Date: June 08, 2020

DOI: $10.23880 /$ ooaj-16000190

\section{Abstract}

Introduction: Eosinophils are thought to play an important role in the symptomatology and pathophysiology of allergic rhinitis. Blood eosinophilia and tissue eosinophilia are characteristic features of allergic inflammation and asthma, conditions associated with prominent production of Th2 cytokines.

Objective: To correlate the eosinophil count of nasal secretions in clinically diagnosed patients of allergic rhinitis and patients with no nasal complaint.

Methods: Patients were selected on the basis of history and clinical examination and equal numbers of controls were taken. Both were subjected to nasal smear examination for eosinophilia.

Results: Overall, eighty seven percent of nasal smears were positive in various degrees among the cases while only around seven percent of slides were positive for eosinophils in control group.

Conclusion: Nasal smear eosinophilia had a very high specificity and moderately high sensitivity in diagnosing allergic rhinitis and it seems to be a potentially valuable test for allergic rhinitis.

Keywords: Allergic Rhinitis; Nasal eosinophilia; Allergy

Abbreviations: AR: Allergic Rhinits; MG: May Grunwald's; DPX: Dibutylphthalate Polystyrene Xylene; SPSS: Statistical Package for Social Sciences.

\section{Introduction}

Allergic rhinitis (AR) is the commonest immunologic disease and is the commonest chronic disease experienced by humans [1]. Although not as glamorous as its surgical counterparts, the management of allergic rhinitis constitutes a large proportion of the day to day practice of general otolaryngologist. In addition to its primary effect, inhalant allergy of the upper respiratory tract might affect the development and clinical course of other disease states such as sinusitis, otitis media and asthma [2]. Allergic rhinitis occurs in atopic individuals who are exposed to common aeroallergens. It is frequently trivialized by patients and doctors. It remains a common cause of morbidity, social embarrassment and impaired performance either at school or in the workplace [3]. Despite its high prevalence, it is often undiagnosed [4].

It has been estimated that up to $50 \%$ of patients evaluated in otolaryngology practices have some component of inhalant allergy in their presenting complaint [5]. Allergic rhinitis is a common condition, though not life threatening but causes significant morbidity in terms of quality of life. Further, an early diagnosis of allergic rhinitis is essential in order to prevent co-morbid conditions like asthma, otitis media, sinusitis and sleep disorders. A careful medical history including the relation between symptoms and exposure to offending agents and a careful physical examination are the basis in diagnosis, but complementary objective tests might be needed. 


\section{Otolaryngology Open Access Journal}

\section{Objective}

To correlate the eosinophil count of nasal secretions in clinically diagnosed patients of allergic rhinitis and patients with no nasal complaint.

\section{Source of Data}

The study was done at the Out-Patient Clinic of Department of Otorhinolaryngology and Head \& Neck Surgery of KLEs Dr. Prabhakar Kore Hospital \& MRC, Belgaum. There were two study groups. One consisting of the patients suffering from allergic rhinitis and other without any signs and symptoms of allergy.

Study Design: Observational study

Period of study: From 1st January 2010 to 31st December 2010

Sample Size: 30 patients in each group

All patients in the age group of 1050 years presenting with two or more complaints of running nose, blocking of nose, itching and sneezing for less than four days a week or for less than four consecutive weeks were taken as intermittent allergic rhinitis and for more than four days a week and for more than four consecutive weeks were taken into persistent allergic rhinitis groups. An equal number of controls who presented without any nasal complaints, without any history of allergy and not suffering from any chronic illness were selected for comparison after age and sex matching. Those who were diagnosed as having vasomotor rhinitis or on antiallergic treatment were excluded from the study.

\section{Methodology}

Nasal swab taken by scraping the mucous membrane of the inferior turbinate using a sterile air dried cotton applicator and smear was made on a glass slide. The slide was stained with May-Grunwald and Giemsa stain. Staining technique: The nasal smears are fixed in methanol for $10 \mathrm{~min}$. May-Grunwald's (MG) stain is diluted with an equal part of phosphate buffer (or tap water). Also, Giemsa stain is diluted with 9 parts of phosphate buffer for 10-15 min. The diluted M.G. stain is poured on the smear. After 5 minutes, the stain is removed and Giemsa Stain is poured. After a further period of 5 minutes, it is washed with and wait for 5 minutes phosphate buffer ( $\mathrm{pH}$ 6.8). The smear is later air dried and mounted on Dibutylphthalate Polystyrene Xylene (DPX).

The percentage of eosinophils per hundred leucocytes are calculated and compared in both the groups.

\section{Statistical Analysis}

Statistical Package for Social Sciences (SPSS) Software was used for data analysis.

Percentage increase in two groups were compared with ChiSquare test.

\section{Results}

Most of the cases were upto the age of 30 years $(80 \%)$. The mean age of the study group was 24.43 while that of the control group was 23.53. In the study group 16 patients $(63.33 \%)$ were males and 14 patients (46.67\%) were females. The same sex ratio was there in the control group. There were 17 patients (56.67\%) with Intermittent Allergic Rhinitis and 13 patients (43.33\%) with Persistent Allergic Rhinitis. Majority of patients $24(80 \%)$ had the eosinophil count in nasal smears more than $10 \%$ ( the value considered to be significant to represent AR) as seen in Table 1. 16 patients(53.33\%) were having watery nasal discharge while 14 patients(46.67\%) were having mucoid nasal discharge. The colour of the nasal mucosa was also noted. 16 patients $(53.33 \%)$ were having dull red color, 10 patients $(33.33 \%)$ were having pale color and 4 patients $(13.33 \%)$ greyish blue color mucosa. The study group was strongly associated with nasal smear eosinophilia. The Chi- Square value -42.000 and "p" value $-0.000+(<0.05)$ was statistically very significant as depicted in Table 2. Also, Intermittent AR was found to be associated with a higher nasal smear eosinophilia(13 patients among cases as opposed to 11 among the control group in the range of $11-50 \%$ ). The Chi square value was 8.668 and "p" value was $0.013(<0.05)$ which is statistically significant (Table 3). In this study, watery type of nasal discharge was observed to be associated more with nasal smear eosinophilia as seen in Figures 1 \& 2. The Chi square value was 10.102 and "p" value was $0.006(<0.05)$ which is statistically significant. However, no association was found between the color of nasal mucosa and nasal smear eosinophilia.

\begin{tabular}{|c|c|c|c|}
\hline Eosinophil \% in nasal smears & Grades & No. of cases & \% \\
\hline$<5 \%$ & I & 2 & $13.33 \%$ \\
\hline $6-10 \%$ & II & 21 & $7.67 \%$ \\
\hline $11-50 \%$ & III & 3 & $10 \%$ \\
\hline$>50 \%$ & IV & 30 & $100 \%$ \\
\hline
\end{tabular}

Table 1: Frequency Distribution of Nasal Smears for Eosinophilia. 


\section{Otolaryngology Open Access Journal}

\begin{tabular}{|c|c|c|c|}
\hline No. of eosinophils & Interpretation & Cases & Controls \\
\hline$<5 \%$ & Normal & 4 & 28 \\
\hline $6-10 \%$ & Doubtful & 2 & 2 \\
\hline $11-50 \%$ & Pathological & 21 & 0 \\
\hline$>50 \%$ & Pathological & 3 & 0 \\
\hline
\end{tabular}

Table 2: Comparison of Eosinophil Count in Study Group and Control Group.

\begin{tabular}{|c|c|c|c|c|c|}
\hline & \multicolumn{5}{|c|}{ Eosinophilia in nasal smear } \\
\hline Disease Type & $<\mathbf{5 \%}$ & $\mathbf{6 - 1 0 \%}$ & $\mathbf{1 1 - 5 0 \%}$ & $>\mathbf{5 0 \%}$ & Total \\
\hline Intermittent & 0 & 0 & 10 & 3 & 13 \\
\hline Persistent & 4 & 2 & 11 & 0 & 17 \\
\hline Total & 4 & 2 & 21 & 3 & 30 \\
\hline
\end{tabular}

Table 3: Nasal Smear Eosinophilia v/s Disease Type.

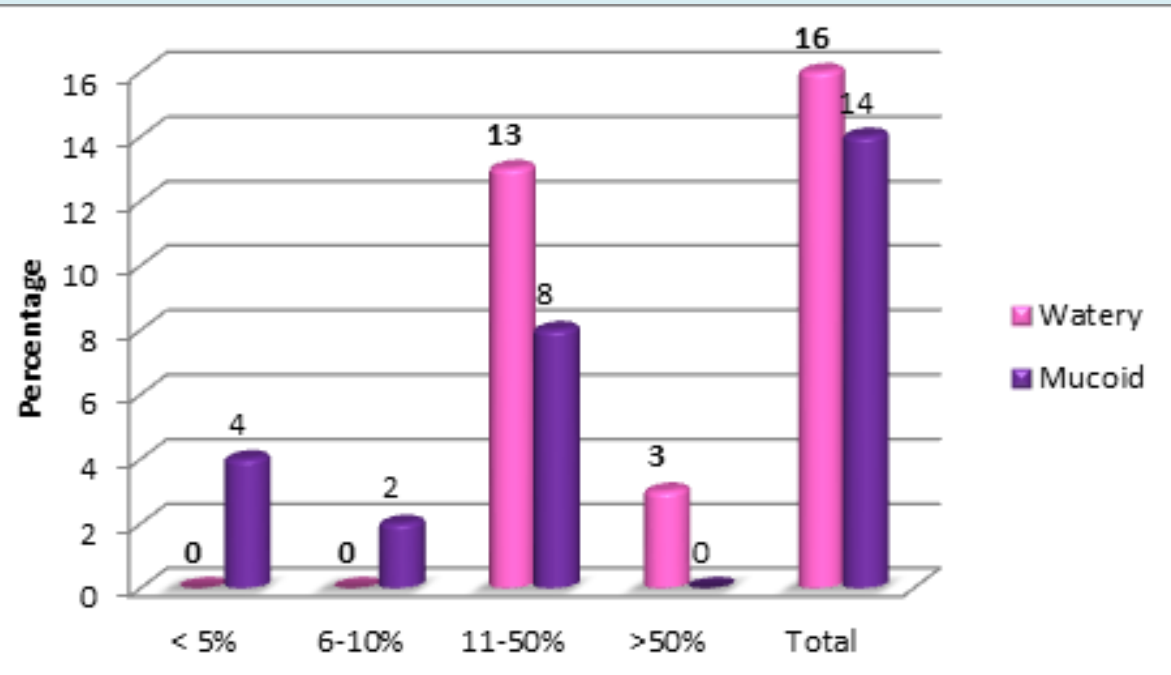

Figure 1: Graph showing Nasal Smear Eosinophilia v/s Type of Nasal Discharge.

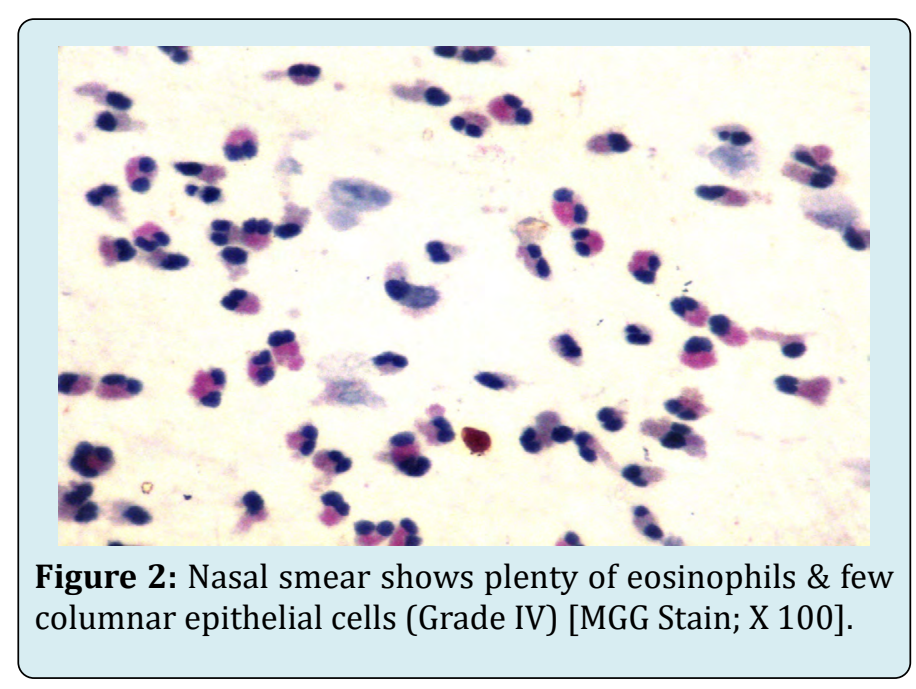

\section{Discussion}

The correlation between clinical allergy and nasal smear eosinophilia was first emphasized by Sood A [6]. It is a generally known fact that in the various shock organs or tissues in which the lesions of allergy occur, the pathological picture is characterized by edema and eosinophilic infiltration. Allergic reactions in the target tissue cause the liberation of various pharamacologically active chemical mediators [6] including the factor for anaphylaxis [Eosinophilic Chemotactic Factor-A (ECF-A)] which leads to the congregations of eosinophil leucocytes in the local sites. The Th-2 cytokine IL-5 is a central factor mediating eosinophil expansion, priming, recruitment and prolonged tissue survival in response to allergic stimuli. IL-5 is synthesized predominantly by Th-2 lymphocytes, 


\section{Otolaryngology Open Access Journal}

but in smaller amounts by mast cells and eosinophils. IL-5 promotes differentiation of terminally committed human and murine eosinophil precursors in bone marrow [7].

While correlating among the intermittent and perennial type of allergic rhinitis, there was a significant association between the intermittent type and nasal smear positivity for eosinophilia. This observation was in concordance with the observation by Canakcioglu S, et al. [8] who noticed rise in nasal eosinophilia during the spring and summer seasons in patients of allergic rhinitis, which are the common months of affection by the disease. Also in many other studies nasal eosinophilia is found to be associated more to seasonal allergic rhinitis as observed in the work done by Goel $\mathrm{U}$, et al. [9]. In a study by Crobach M, et al. [10] was concluded that the sensitivity of nasal smear eosinophilia for allergic rhinitis was $18 \%$ and the specificity was $96 \%$. Possible explanations include firstly, many of those patients did not have symptoms at the moment they consulted their general practitioner. As eosinophilia is correlated with exposure to allergens it has been recommended that asymptomatic patients are asked to return when they experience symptoms. Moreover, to obtain higher sensitivity it has been advised that three smears are taken on separate occasions by Mygind.

Secondly, nasal eosinophilia is negatively influenced by viral or bacterial infections [11]. Thirdly, the use of topical corticosteroids reduces the percentage of eosionophils. Some patients in the study were using topical corticosteroids. In the study by Sood A [6] 80\% of the nasal smears were positive in various degrees for eosinophils while in the control group only $5 \%$ showed any kind of positivity. The results of nasal smear eosinophilia were compared with skin prick test. The percentage of correlation between the nasal smear eosinophilia and skin test came out around $88 \%$ and further substantiated the diagnosis of allergic rhinitis. In our study the smear positivity was found in $86.67 \%$ of cases and $6.67 \%$ of the controls (in the doubtful range). The cause for this has been discussed by Malmberg and Halopainen [11]. They state that an infection may cause a small number of eosinophils to appear in the nasal secretion of non-allergic persons. And also conversely it may cause a temporary disappearance of the eosinophils from the secretions of persons with allergic rhinitis [6].

In view of the results of our study and many others discussed before which state nasal eosinophilia to be a highly specific and moderately sensitive test and as stressed by Miri S, et al. [12], this highly valid test can be quickly and easily performed and read. And being an in-expensive test, it can be used to screen the patients of allergic rhinitis. In his study, nasal smear eosinophilia had a specificity of $96 \%$ and sensitivity of $62 \%$. This seemed to be a potentially valuable test for allergic rhinitis. In a study done by Canakcioglus, et al. [8], it was found that nasal eosinophilia in AR significantly increased during the spring and summer seasons. They concluded that, the presence of nasal eosinophilia strongly supports the diagnosis of AR. Also a significant reduction or disappearance of inflammatory cells in cytologic samples can confirm the resolution of the pathologic condition during the medical treatment.

Few data is available on the natural history of NAR, including whether it progresses to AR [13]. In a recent study, a group of 180 Non AR subjects diagnosed during 2000-2004 and re-evaluated in 2007 demonstrated de novo sensitization to aeroallergens in $24 \%$ of patients as assessed by Skin Prick Test (SPT), serum IgE or both [13]. These results suggest that Local AR may be part of AR with a positive SPT result at the beginning of the natural course of disease. This concept further enhances the value of nasal cytology as it can detect the cases in early stage of natural course of disease.

A study by Bakshaee $M$, et al. [14] showed that smear eosinophilia is more sensitive and specific than nasal biopsies for the detection of allergic rhinitis. The results of nasal smear eosinophilia in this study are comparable with most of the studies mentioned. Matching of nasal eosinophilia with the type of nasal discharge showed watery type of nasal discharge to be associated with nasal smear eosinophilia. The results were found to be statistically significant $(p<0.05)$. In another study by Sood A [6] the type of nasal discharge was found to be of borderline significance. In that study also watery type of discharge was more associated to higher percentage of eosinophils in the samples. Also Manners BTB [15] found high positivity of nasal smear eosinophilia in patients with profuse rhinorrhoea. Also no significant link was found between the nasal smear eosinophilia and the colour of nasal mucosa, thereby meaning they were independent of each other. This finding was in agreement with a study by Sood A [6].

\section{Conclusion}

In this study it is observed that, a strong association of nasal smear eosinophilia with allergic rhinitis exists which is in concordance with many other studies. Those patients with Intermittent type of allergic rhinitis and cases with watery type of nasal discharge were found to be associated with nasal smear eosinophilia and the association was found to be statistically significant. However, in light of other studies which have compared nasal smear for eosinophilia with skin prick test, nasal smear for eosinophilia is found to be highly specific and moderately sensitive to diagnose allergic rhinitis and hence, we recommend this test to screen as well as to diagnose allergic rhinitis. Also, being a noninvasive and inexpensive Out Patient based test, it can also be used to monitor the response to the treatment given. It is 


\section{Otolaryngology Open Access Journal}

of paramount importance to know that with newer concepts emerging in the management strategy of allergic rhinitis, it is punitive to come up with lesser invasive and easily applicable methods such as nasal smear for eosionphilia to easily screen, diagnose and monitor the patients during the follow up period in order to explore newer domains in the treatment protocol of allergic rhinitis.

\section{References}

1. Kaliner MA (1993) Allergic Rhinitis. In: Mygind N and Naclerio RM (Eds.), Allergic and non-allergic rhinitis clinical aspects, Philadelphia: W.B. Saunders Company, pp: 153.

2. Mabry RL (1998) Allergic Rhinitis. In: Cummings CW, et al. (Eds.), Otolaryngology Head and Neck Surgery. $3^{\text {rd }}$ (Edn.), Missouri, USA; Mosby, pp: 902-908.

3. Durham SR (1997) Mechanisms and treatment of allergic rhinitis. In: Kerr AG (Eds.), Scott-Brown's otolaryngology. $6^{\text {th }}$ (Edn.), Oxford: Butter worth. Heinemann, 4.

4. Skoner DP (2001) Allergic rhinitis: definition, epidemiology, pathophysiology, detection, and diagnosis. J Allergy Clin Immunol 108(1): 2-8.

5. Krouse JH, Robert J Stachler, Anand S (2003) Current in vivo and in vitro screens for inhalant allergy Otolaryngol Clin North Am 36(5): 855-868.

6. Abhey S (2005) Diagnostic significance of nasal eosinophilia in allergic rhinitis. Indian J Otolaryngol Head Neck Surg 57(1): 13-16.

7. Rosenberg HF, Phipps S, Foster PS (2007) Eosinophil trafficking in allergy and asthma. J Allergy Clin Immunol 119(6): 1303-1312.
8. Canakcioglu S, Tahamiler R, Saritzali G, Alimoglu Y, Isildak $\mathrm{H}$, et al. (2009) Evaluation of nasal cytology in subjects with chronic rhinitis: A 7-year study. Am J Otolaryngol 30(5): 312-317.

9. Urmil G, Bazaz-Malik G, Mohindra SK (1984) Significance and comparison of blood, nasal secretion and mucosal eosinophilis in nasal allergy. Indian J Pathol Microbiol 27(1): 27-32.

10. Crobach M, Hermans J, Kaptein A, Ridderikhoff J, Mulder J (1996) Nasal smear eosinophilia for the diagnosis of allergic rhinitis and eosinophilic non-allergic rhinitis. Scand J Prim Health Care 14(2): 116-121.

11. Malmberg H, Halopainen E (1979) Nasal smear as a screening for immediate-type allergy. Allergy 34(5): 331-337.

12. Miri S, Farid R, Akbari H, Amin R (2006) Prevalence of allergic rhinitis and nasal smear eosinophilia in 11 to 15 yr old children in Shiraz. Pediatr Allergy Immunol 17(7): 519-523.

13. Rondon C, Fernandez J, Canto G, Blanca M (2010) Local allergic rhinitis: concept, clinical manifestations and diagnostic approach. J Investig Allegol Clin Immunol 20(5): 364-371.

14. Bakhshaee M, Fereidouni M, Farzadnia M, Varasteh AR (2010) The nasal smear for eosinophils, it's value and it's relation to nasal mucosal eosinophilia in allergic rhinitis. The Iranian Journal of Otorhinolaryngology 22(60): 7378.

15. Manners BTB (1974) The diagnostic value of detecting eosinophilia of nasal secretions in allergic rhinitis. J R Coll Gen Pract 24(143): 397-399. 